\title{
A Linux-Based Integrated Structural Health Monitoring System for Bridges in Remote Regions
}

\author{
Yi Yan ${ }^{1 *}$, Zhangyong $\mathrm{Wu}^{2}$, Xuantao $\mathrm{Wu}^{3}$, Xuanlin Zhou ${ }^{4}$, Chengxian Weng ${ }^{5}$ \\ ${ }^{1}$ Chongqing Jiaotong University, Chongqing 400074, China \\ ${ }^{2}$ Shanxi Hairong Engineering Test \& Testing Co., Ltd., Chongqing Branch, Chongqing 401122, China \\ ${ }^{3}$ Chongqing Zhengda Engineering Consulting Co., Ltd., Chongqing 401122, China \\ ${ }^{4}$ Chongqing Jiaotong University Engineering Design and Research Institute Co., Ltd., Chongqing 400074, China \\ ${ }^{5}$ T.Y.Lin International Engineering Consulting (China) Co., Ltd., Chongqing 401121, China
}

Corresponding Author Email: dingyc@mails.cqjtu.edu.cn

https://doi.org/10.18280/i2m.180603

Received: 15 March 2019

Accepted: 28 September 2019

\section{Keywords:}

structural health monitoring (SHM),

Linux, opensource, integrated design

\begin{abstract}
This paper aims to design a stable, reliable, energy-efficient yet low-cost structural health monitoring (SHM) system for bridges in remote regions. First, the integrated system design was adopted based on the Linux architecture, and the power-efficient opensource hardware was taken as the mini Linux PCs on the bridge site. Next, several sensor subsystems were integrated physically via the Ethernet, while nine core software modules were developed based on opensource software and integrated into a logical whole. The stability of the system was improved through dual PC redundancy and intelligent fault diagnosis. The data collected by sensors were analyzed in real time and only the valid data and alarms were transmitted to the remote monitoring center. In addition, the remote transmission and active control were completed, with the aid of $3 \mathrm{G} / 4 \mathrm{G}$ router, VPN and reverse SSH tunneling. In this way, the author developed an easy-to-maintain and cost-efficient SHM system. Finally, the effectiveness of the system was verified through the application in a bridge on an expressway in Yunnan Province, China. The results fully demonstrate the robustness, accuracy and cost effectiveness of the proposed system. This research fills up the gap in Linux-based SHM system design for bridges in remote regions, providing a reliable and advanced reference for the construction of bridge SHM systems.
\end{abstract}

\section{INTRODUCTION}

Bridge structure is an important component of lifeline engineering. The recent boom in bridge construction calls for operation security of bridges. However, it is a challenging task to maintain the healthy operation of large-span bridges. The current structural health monitoring (SHM) programs for bridges mainly rely on smart sensors and reliability and security evaluation. The design of the monitoring system directly bears on the implementation effect. The key to the system design lies in the physical and logical integration between sensor subsystems with different functions, which lays the basis for the central control of data acquisition, storage, preprocessing and remote transmission of all sensors [1-4].

In remote regions, the bridges generally work under harsh environment: the lack of sufficient power supply and stable communication network, as well as the inconvenience and high cost of maintenance. Against this backdrop, nonconventional sources of energy, such as the solar power, need to be utilized to provide enough electricity, support stable data processing and enable robust control of the integrated monitoring system.

Depending on the mode of data acquisition, the conventional integrated SHM systems fall into two categories: the distributed acquisition system and the centralized acquisition system. In the former system, the raw data are collected by various types of acquisition instruments, ranging from data acquisition card [5], fiber Bragg grating (FBG) sensor [6], DataTaker [7] to OpenDAQ [8]. The collected data are transmitted to a dedicated personal computer (PC) or workstation [9] through a remote transmission model or via hardware interfaces like USB, serial ports and RJ45 sockets [10]. The PC runs one or more monitoring software on Windows, such as LabVIEW [1,8] and SCADA [9], to name but a few.

In the latter system, the raw data are directly collected by a professional acquisition module and transmitted to the monitoring center via the communication module [11-15]. Typical examples of professional acquisition modules include embedded wireless data acquisition system with functions of data terminal unit (DTU) and general packet radio service (GPRS), and the remote terminal unit (RTU) based on Embedded Linux. However, the centralized acquisition system fails to preprocess or analyze the collected data in real time.

Over the years, many scholars have attempted to improve the design of integrated SHM systems. For instance, Boller [16] introduced the concept of the SHM to aircraft design, compared the established load monitoring program with an emerging damage monitoring solution, and described how to select the technique for damage monitoring. Zaurin and Catbas [17] proposed to integrate video images and sensor data for the SHM, and demonstrated the potential of the integrated method in security monitoring of civil structures, especially bridges. Based on Bayesian probability, Ling and Mahadevan [18] predicted fatigue damages using both online and offline SHM data. King et al. [19] reviewed the data fusion techniques and 
algorithms that help to interpret the data of wearable sensors in SHM apps. Daroogheh et al. [20] combined computational intelligence and model-based design (MBD) into a novel hybrid architecture for the prediction and SHM of nonlinear systems. Bae et al. [21] experimentally evaluated the monitoring effect of wireless sensor network (WSN) of a bridge structure in the obstructed environment. Wang [22] applied the WSN in the SHM of bridges. Vanzwol et al. [23] implemented long-term SHM of the Crowchild Trail Bridge. Ogundipe et al. [24] performed the wavelet transform (WT) on the data collected from the Machang Bridge, a cable-stayed bridge in South Korea. Venglar et al. [25] developed an SHM system for a port bridge.

Nevertheless, the existing bridge SHM systems cannot be directly applied to bridges in remote regions, due to their high electricity consumption and maintenance cost. Moreover, there is still an ample room to improve the stability, reliability and effectiveness of these systems. To solve these problems, this paper proposes an integrated SHM system for bridges in remote regions based on the Linux, using opensource hardware and software.

The remainder of this paper is organized as follows: Section 2 introduces the overall design of the integrated SHM system; Section 3 details the software of the proposed system; Section 4 applies the system to monitor an in-service bridge on an expressway in Yunnan Province, China, and verifies the stability and reliability of the system; Section 5 puts forward the research conclusions.

\section{OVERALL DESIGN}

\subsection{Design considerations}

Despite the robustness of existing integration techniques, special attention should be paid in the following aspects in the design of an SHM system for bridges in remote areas.

(1) System stability. The SHM system needs to monitor the bridge structure continuously, from the construction phase to the operation phase. In remote regions, the system is influenced by a variety of factors, some of which are very rare. Therefore, it is an important task to ensure the stable operation of the SHM system.

(2) Data reliability. The monitoring data captured by sensors are easily accumulated, posing a heavy burden on the server side of the monitoring center. To solve the problem, the invalid data and abnormal data should be preprocessed before uploading to the monitoring center.

(3) Fault diagnosis. The timely detection of faults is critical to the normal operation of the SHM system. The system must be able to identify the faults onsite and alert operators in a timely and visual manner. Anti-theft cameras should be installed to prevent intrusion.

(4) Cost saving. The remoteness of the bridge pushes up the cost of maintenance. To save cost, the SHM system should support remote control, allowing offsite operators to modify parameters, debug software and control sensors.

\subsection{Overall framework}

Considering the above factors, the author decided to develop a high-performance SHM system for bridges in remote regions based on Linux, rather than Windows. At present, most integrated SHMs for bridges use industrial PCs based on Windows Server. However, this operating system is not stable in the long run and incurs a high cost. By contrast, the Linux is an open-source operating system, which is free, stable and reliable.

Thanks to the maturity of opensource hardware, this paper only needs to emphasize on the software development based on Linux. With the aid of opensource hardware, the physical integration was realized with some sensor subsystems via the Ethernet, while the logical integration was achieved through software modules designed based on opensource software. Figure 1 illustrates the framework of the Linux-based integrated SHM system for bridges in remote regions, where DI and DO stand for data input and data output, respectively.
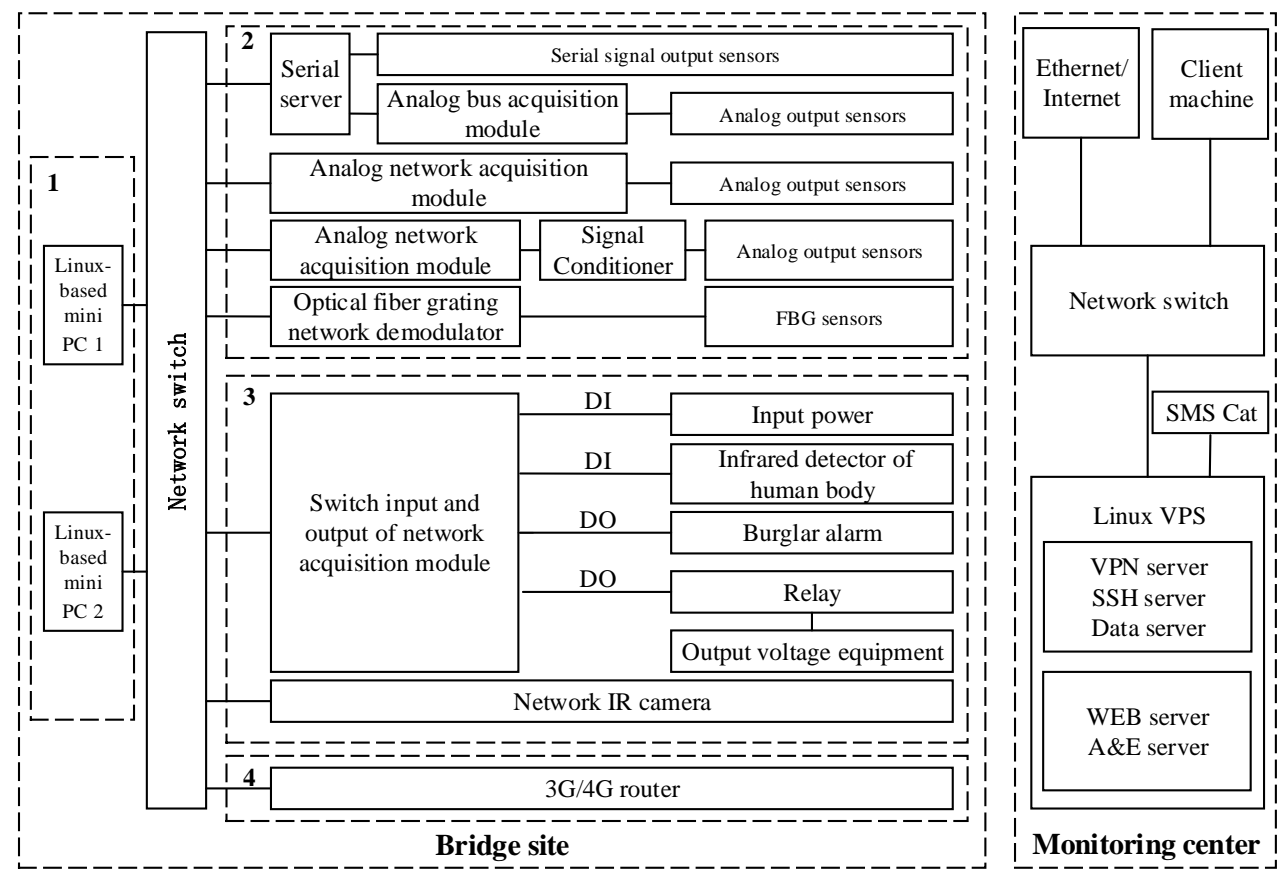

Figure 1. The framework of the integrated SHM system 


\subsection{Bridge site}

As shown in Figure 1, the integrated system consists of two parts, namely, the bridge site and the monitoring center. The bridge site can be further divided into the following four subparts.

(1) Linux-based mini PCs. The mini PCs are the central processing unit (CPU) of the entire system. They are responsible for acquiring, storing, processing and transmitting data, as well as the coordination and control of all subsystems. The most popular Linux-based mini PCs include CubieBoard/CubieTruck, Raspberry Pi, BeagleBone and PCDuino. There are multiple advantages of these mini PCs, including small volume (card size), low power consumption $(<5 \mathrm{~W})$, high cost performance (high-performance CPU and memory at less than RMB 1,000 yuan), and fast switching speed. In addition, they are provided with a hardware watchdog. Two Linux-based mini PCs were deployed in the system to provide the redundancy needed to ensure system reliability and cope with $\mathrm{PC}$ failure.

(2) Parameter monitoring equipment. Multiple sensors and data acquisitors were adopted to collect data and transmit them to the PC via the Ethernet. Among them, the serial server converts the serial port (RS232/RS485) signals from/to network data, such that the mini PC can receive all the output of serial signal output sensors via sockets. The standard analog signals (4-20mA and $0-5 \mathrm{~V})$ are transmitted to the PC through bus or analog network acquisition module; the nonstandard analog signals are converted into the standard form by the signal conditioner, before being sent to the PC; the signals outputted by the FBG sensors are demodulated and then transmitted to the $\mathrm{PC}$ via the Ethernet.

(3) Intelligent fault diagnosis. This subpart mainly encompasses the network acquisition module of switch input and output, switch signal equipment, network infrared camera, etc. Whenever the solar energy is inputted with no voltage, this subpart will be activated to judge the voltage state, in case the normal operation of field equipment is affected by charging failure. To prevent theft, the infrared human body detector issues a warning sound and takes a photo of any approaching personnel, and transmit the photo to the monitoring center. If a field device crashes in continuous operation, it can be restarted by the remote control of relay. Once an accident occurs in the field, the cameras will automatically start to take photos of the scene.

(4) Remote communication. In this subpart, a $3 \mathrm{G} / 4 \mathrm{G}$ router provides direct access to the Internet, facilitating the real-time transmission of massive data and remote control of active access. The network operator will assign a private IPv4 address to the terminal router. The private IPv4 address is selected because of two reasons: public IPs are only assigned by the Telecom $3 \mathrm{G} / 4 \mathrm{G}$ network in a few Chinese provinces; the public IPs are quickly depleting under the IPv4 framework. Then, the VPN and reverse SSH tunneling were adopted to enable the remote access of private IP to the PC.

\subsection{Monitoring center}

The monitoring center is mainly responsible for data reception and storage, security assessment, early warning, etc. Many of these operations induce a heavy computing load and may interfere in the operation of other software. Therefore, different servers were provided to realize the multiple functions. The main components of the monitoring center are as follows:

(1) Linux VPS. The VPS (virtual private server), such as VMware ESXi and Ubuntu+VirtualBox, is a virtualization technique based on the cloud platform. This technique was adopted in the monitoring center to realize at least two virtual PCs on one physical server: (a) VPN Server, SSH server and data server; (b) Web server, assessment and early warning (A\&E) server.

(2) Other components. Other components are needed to connect the physical server to the network switch, client, and the Internet, giving it a fixed IP or DNS domain name. For example, an SMS Cat was installed to send the A\&E results vi SMS or MMS.

\section{SOFTWARE DESIGN}

The software is the cornerstone of the integrated SHM system. On the bridge site, the software, the software needs to direct, call and drive the operations, data interaction and communication of each subsystem. In the monitoring center, the software needs to realize security A\&E through reception, storage, analysis, query and display of field data.

In the system, environments like the SSH, VPN and phpMyAdmin are established, using free opensource programming languages (Python and PHP), database software (MySQL) and web server (Apache). The software for the bridge site client and the monitoring center server was developed through modular programming, according to the communication protocol or the API of the sensors and devices.

\subsection{Software design for bridge site client}

Nine core modules were designed for the software of bridge site client.

(1) Remote access module. This module supports the active visits between the client side and the server side, mainly using the VPN and the reverse SSH tunneling.

(a) VPN. The VPN runs in the bridge site client, using a fixed IP address assigned by the client. In this way, any PC or other VPN client on the server LAN can access the bridge site client remotely, using the intranet IP.

(b) Reverse SSH tunneling. On the client side, some ports (e.g. MySQL port 3306, SSH port 22 and web port 80) are mapped to their corresponding ports on the server side, with known DNS domain name (or fixed IP). Hence, the software on the client side can be accessed with the server name and port number, allowing direct modification and debugging of the bridge site client.

Table 1 compares the merits and defects of the two remote access methods.

Table 1. The merits and defects of the two remote access methods

\begin{tabular}{ccc}
\hline $\begin{array}{c}\text { Remote access } \\
\text { methods }\end{array}$ & Merits & Defects \\
\hline VPN & $\begin{array}{c}\text { Supports TCP and UDP } \\
\text { protocols }\end{array}$ & $\begin{array}{c}\text { Only allows } \\
\text { intranet access }\end{array}$ \\
SSH & $\begin{array}{c}\text { Allows accesses from } \\
\text { anywhere via the Internet }\end{array}$ & $\begin{array}{c}\text { Only supports } \\
\text { TCP protocol }\end{array}$ \\
\hline
\end{tabular}

The remote access module combines the VPN with the SSH. This dual mechanism guarantees the stable communication between the client side and the server side. The state of remote 
connection is judged in real time by the remote access module. If necessary, the VPN and the SSH services will be restarted to protect the smoothness of communication. Any change in the network state will be recorded in the network state log of the MySQL database.

(2) Data acquisition module. In the SHM system, all sensors and other acquisition instruments must have a primitive communication protocol or Linux drivers and API. Under this principle, the data acquisition module was developed in Python language through socket programming and UDP-based multithreading. Each valid port or data acquisitor was treated as a sub-thread of the serial server. Then, multiple threads were controlled by the host thread to complete sync acquisition. The module parameters and sensor data are both stored in the MySQL database. The data collected by any sensor are immediately filtered by a limited amplitude. The raw data and the filtered data will be stored in the measured data log of the database. The limited amplitude filtering is a relative fast and effective approach to preprocess the collected data, because of the gentle changes in bridge structure and the rarity of sudden damage. Meanwhile, a secondary thread is enabled to assess if the corresponding parameters in the database have changed. If yes, the variables of the software will be updated in a timely manner.

Furthermore, the field data are collected in a relatively short period by this module. If the data cannot be acquired by sensors for $\mathrm{N}$ times $(\mathrm{N}>3)$, the acquisition failure will be saved in the alarm log of the MySQL database.

(3) Monitoring module. This module mainly collects and control the state of the network acquisition module of switch input and output, assesses the on-off state of solar panels in the field, and intercepts the DI of switch signals triggered by the infrared human body detector. Then, suitable state response will be made by controlling the opening degree of the DO switch, and the current photo will be collected and stored by calling the camera API. Finally, the bridge state will be saved in the alarm log of the MySQL database.

(4) Control and interaction module. This module serves the users who access the $\mathrm{PC}$ in the client/server $(\mathrm{C} / \mathrm{S})$ mode. All the commands from the server side to the client side can be defined and implemented on this module. The UDP protocol is adopted to judge the access right of a user and respond to user operations, including parameter setting/modification, query for operating state and CPU temperature, request restart the PC and some sensors, and request to upload the primitive data.

(5) Alarm and data upload module. Targeting the database, this module covers the following five functions:

a. Data preprocessing. The data collected at each measuring point are filtered. Then, the maximum, minimum, mean and instantaneous values are calculated based on a certain period, and stored in the measured data log of the MySQL database. The period was set to $10 \mathrm{~min}$ between two fixed time points, e.g. 12:00 and 12:10. If the mean value falls outside the valid range of the sensor, the data will be stored in the alarm log.

b. State determination module. The network state log is read by a sub-thread with a polling period shorter than $10 \mathrm{~min}$. Then, the presence of remote connection is judged, and the judgement will be assigned to the global parameters under the module.

c. Remote data transmission. The presence of remote connection is judged by a sub-thread with a polling period slightly longer than $10 \mathrm{~min}$. If there is a remote connection, the identity of the last received data will be queried first in the measured data table. Then, the new field data will be transmitted to the server side under the UDP. The server will sectionally compress the data by methods like 7-Zip, and store the compressed data in the database. This transmission strategy has several advantages. For instance, the server can obtain all the field data even after reconstruction, the transmission process can adaptive to the network state, the data are transmitted rapidly under the UDP, and the $3 \mathrm{G} / 4 \mathrm{G}$ network flow is limited.

d. Alarm upload module. The update in the alarm log is checked by a sub-thread with an even shorter polling period. If the server side is accessed, the latest alarm will be uploaded to the monitoring center under the UDP for segmented compression. If the server side is not visited despite the Internet access, the communication module of $3 \mathrm{G} / 4 \mathrm{G}$ routers will execute the command to send SMS alarms to the responsible person, notifying him/her to handle the fault in a timely manner.

(e) History deletion module. The disk occupancy is queried at fixed intervals to prevent the hard disk from being fully occupied. Once the occupancy surpasses the preset threshold, some of the historical data will be removed. In this module, a control index is set up in the data log to regulate the query rate, such that the data queries will not drag down the performance of the PC.

(6) Web service module. In this module, the PHP technology enables users to perform web-based operations, such as parameter configuration and data query at the bridge site.

(7) Primary watchdog module. This module mainly monitors the resource occupancy of the above six modules, restarts the process of high occupancy, and releases the memory in a timely manner. If the process "auto-switch module of standby system" appears, it will be closed after 10s. Moreover, the PC will restart automatically at a certain interval.

(8) Secondary watchdog module. This module monitors the resource occupancy of the primary watchdog, providing a further guarantee to system stability.

(9) Auto-switch module of standby system. The primary PC and the standby $\mathrm{PC}$ have the same initial system configuration. The only difference is that the primary PC runs more than 8 modules, while the standby PC only runs one module. This module checks the necessity to access the primary $\mathrm{PC}$ from the standby PC, and to sync their databases. If the primary PC suffers from hardware failure, the first 8 modules of the PC will be started immediately, and the failure state will be timely recorded in the alarm log. In addition, an SMS will be sent to the responsible person, asking him/her to take countermeasures.

\subsection{Software design of monitoring center server}

According to the system design, the server side include two Linux-based virtual PCs, which perform different functions using the same database. The software design on the server side is detailed below.

(1) Software design for VPN, SSH and data servers. The servers are configured in SSH, VPN, MySQL, Apache and PHPMyAdmin environments. The data interception, acquisition and storage function are written in Python.

(2) Software design for web server and A\&E server. This virtual server mainly achieves functions like parameter setting, artificial detection of data entry, data display, and web-based 
security A\&E. These functions are completed by the PHP and the corresponding framework. To reduce the computing complexity, the security A\&E function is implemented in Python. Upon detecting any failure or security hazard, the SMS Cat will send the responsible person an alarm in the form of SMS or MMS. The alarm can also be sent via third-party apps like WeChat. The data are displayed in the form of curves, charts, etc.

\section{ENGINEERING APPLICATION}

\subsection{Application procedure}

To verify its effectiveness, the proposed SHM system was applied to monitor the state of a $452.88 \mathrm{~m}$-long three-span continuous girder rigid-frame bridge on an expressway in Yunnan Province, China. The three spans are respectively $77 \mathrm{~m}$, $140 \mathrm{~m}$ and $77 \mathrm{~m}$. The continuous girder includes $5 \times 30 \mathrm{~m}$ segments. Due to its remote location, the bridge is not connected to any power grid or wired network. The only communication available at the site is Telecom $3 \mathrm{G}$ network. Each box girder is connected to the pier and abutment on the two ends, respectively, and can only accessed by a bridge inspection vehicle.

The maintenance department of the bridge has been commissioned to monitor the permanent deformation of the main bridge for three years and maintain the bridge afterwards. Considering the abundance of solar energy, the proposed integrated SHM system was adopted to monitor the bridge health, and collect valid data through the monitoring period.
The system was implemented in the following steps:

Step 1: The entire system is powered by a $200 \mathrm{ah} 24 \mathrm{~V}$ photovoltaic (PV) module. The core sensors were installed at the measuring points inside each box girder. To facilitate later maintenance, the other devices were deployed near the abutment outside the box girder.

Step 2: The opensource hardware CubieTruck was adopted to control the PC. The hardware has a dual core ARM CPU (1GHz), a 2G DDR3 memory, a 32GB solid state disk (SSD), and a watchdog. The power consumption of the hardware is less than $5 \mathrm{~W}$. The Lubuntu Server, a lightweight operating system, was installed by CubieTruck, providing the operating environment for the software.

Step 3: The permanent deformation of the bridge was measured by the reliable strategy of hydrostatic levelling. With the help of a bridge inspection vehicle, the operators entered the box girder, and installed the hydrostatic level sensor and temperature sensor at segment $0 \#$ (the reference point), 1/4 span, midspan and 3/4 span of the main span. Then, the sensors were connected to the integrated control box outside the box girder via the RS485 bus. Moreover, the end of the communicating pipe was fixed to the bridge deck, making it easy to add water during later maintenance. Figure 2 presents some of the devices installed on the site.

Step 4: With VPN and web access functions, the industrial grade Telecom 3G router (model: H7921) and its supporting antenna were installed to provide access to the Internet. The VPN IP allocated by the server is 192.168.31.18. Figure 3 shows the interface of the remote control terminal that enables the Internet access of the bridge site client.

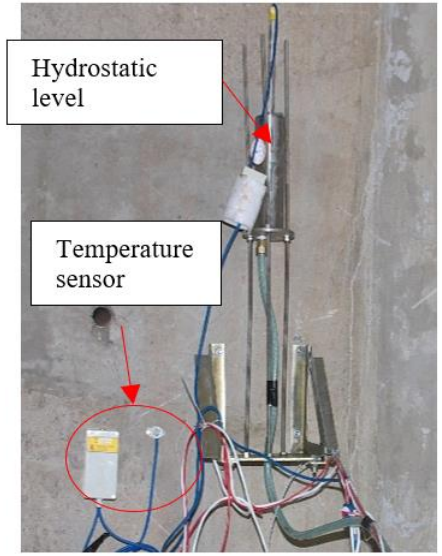

(a) Reference point

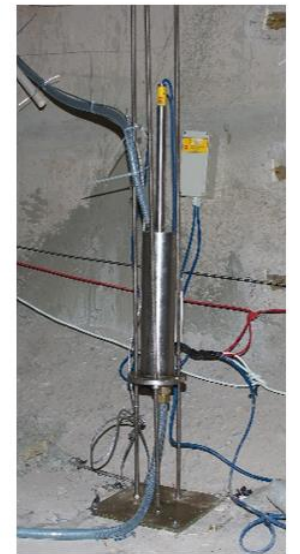

(b) Midspan measuring point

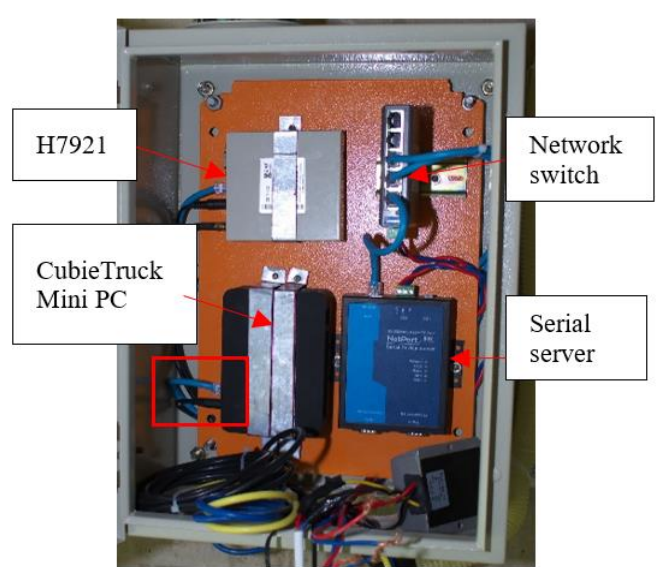

(c) Integrated control box

Figure 2. Some of the devices installed on the site

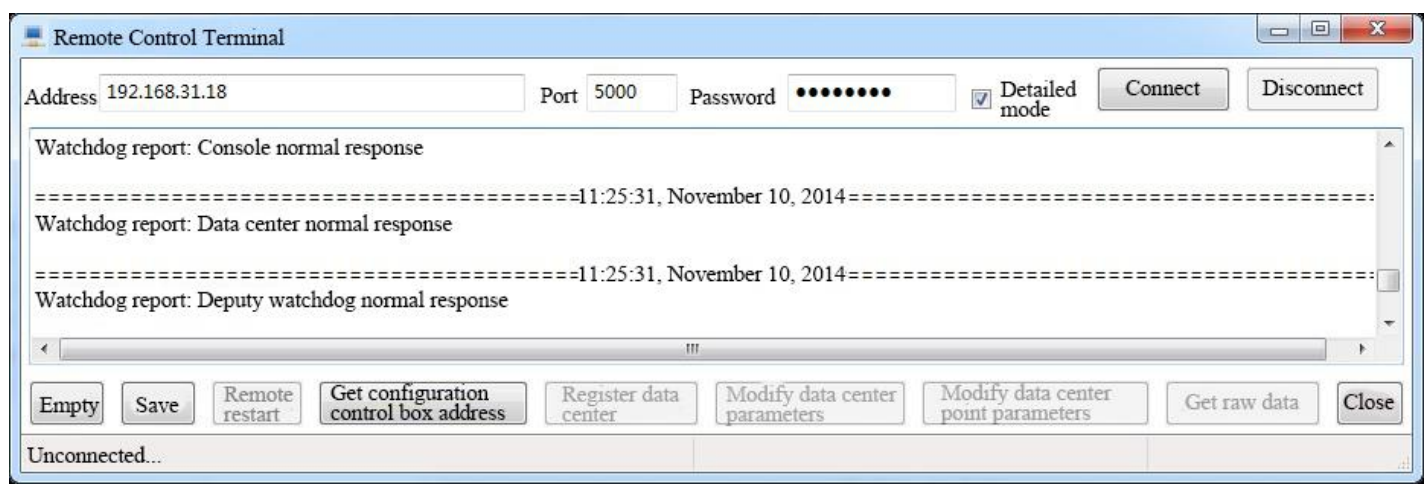

Figure 3. The interface of remote control terminal 
Step 5: Combined with the R-SSH command, the autossh software was employed for the reverse tunneling between the main ports of the client and the server. The mapping between key ports is explained in Table 2 below.

Table 2. Port mapping for reverse SSH tunneling

\begin{tabular}{ccc}
\hline $\begin{array}{c}\text { Device name and } \\
\text { port number }\end{array}$ & $\begin{array}{c}\text { Server } \\
\text { port }\end{array}$ & Note \\
\hline H7921: 10086 & 10020 & H7921 web access \\
PC: 22 & 10021 & SSH remote control \\
PC: 3306 & 10022 & MySQL database access \\
PC: 80 & 10023 & PC web access \\
\hline
\end{tabular}

Step 6: The server was deployed in a professional computer room and assigned a fixed DNS domain name. The data acquisition cycle was set to $5 \mathrm{~s}$, and the maximum, minimum, mean and instantaneous values were analyzed statistically every $10 \mathrm{~min}$. In the end, only the statistical results and alarms were uploaded to the monitoring center. However, the raw data could be acquired by active remote queries.

\subsection{Results and analysis}

The monitoring effect of bridge deflection was analyzed with the midspan data as the representative. Figure 4 shows the time history of the difference between the hydrostatic level and the reference value at the midspan of the right span. Note that the negative sign means the measuring point is higher than the reference point; the lower the negative value, the higher the position of the measuring point; the change of the difference relative to the initial time is the bridge deflection.

Figure 5 provides the time history of midspan defection and temperature recorded by the system from July 24, 2014 to April 24, 2015. Note that the positive and negative signs stand for down-warping and up-warping, respectively.

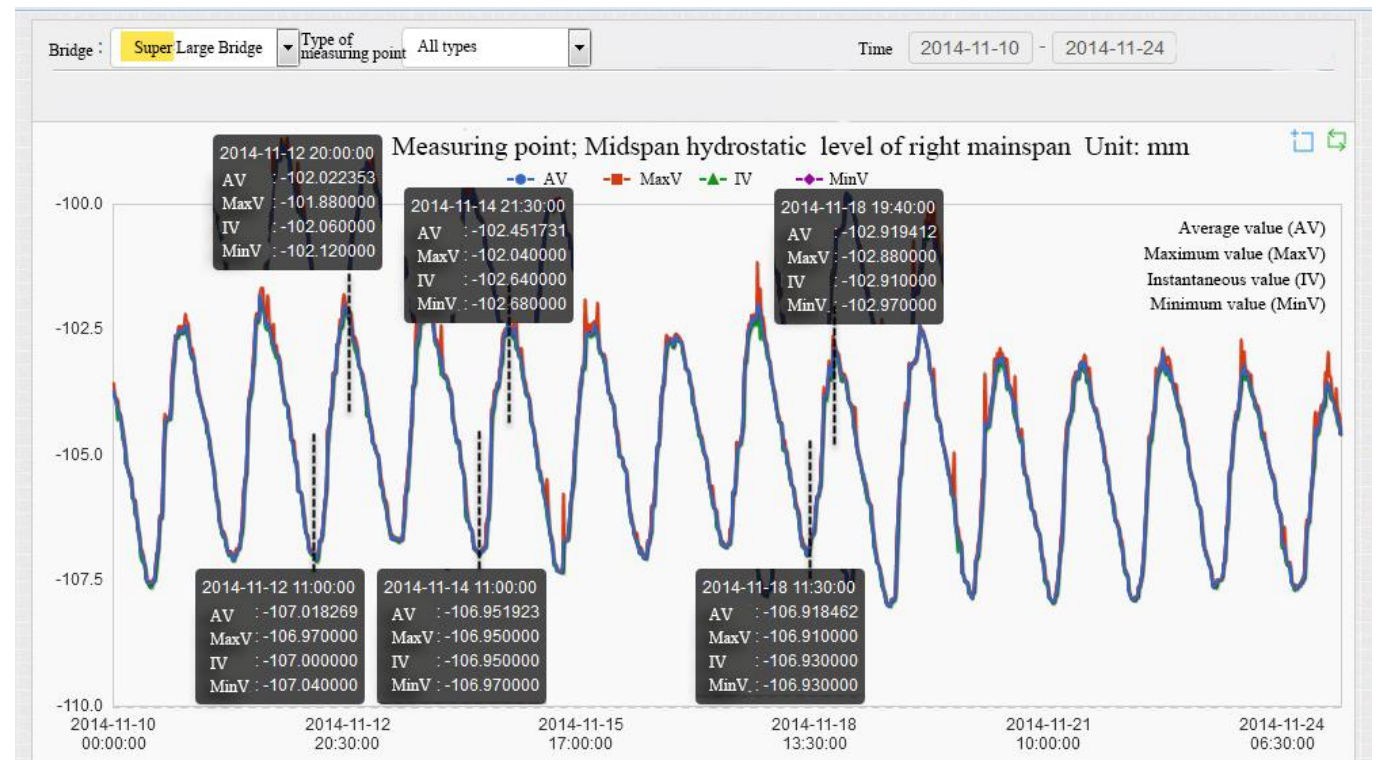

Figure 4. Time history of the difference between the hydrostatic level and the reference value at the midspan of the right span

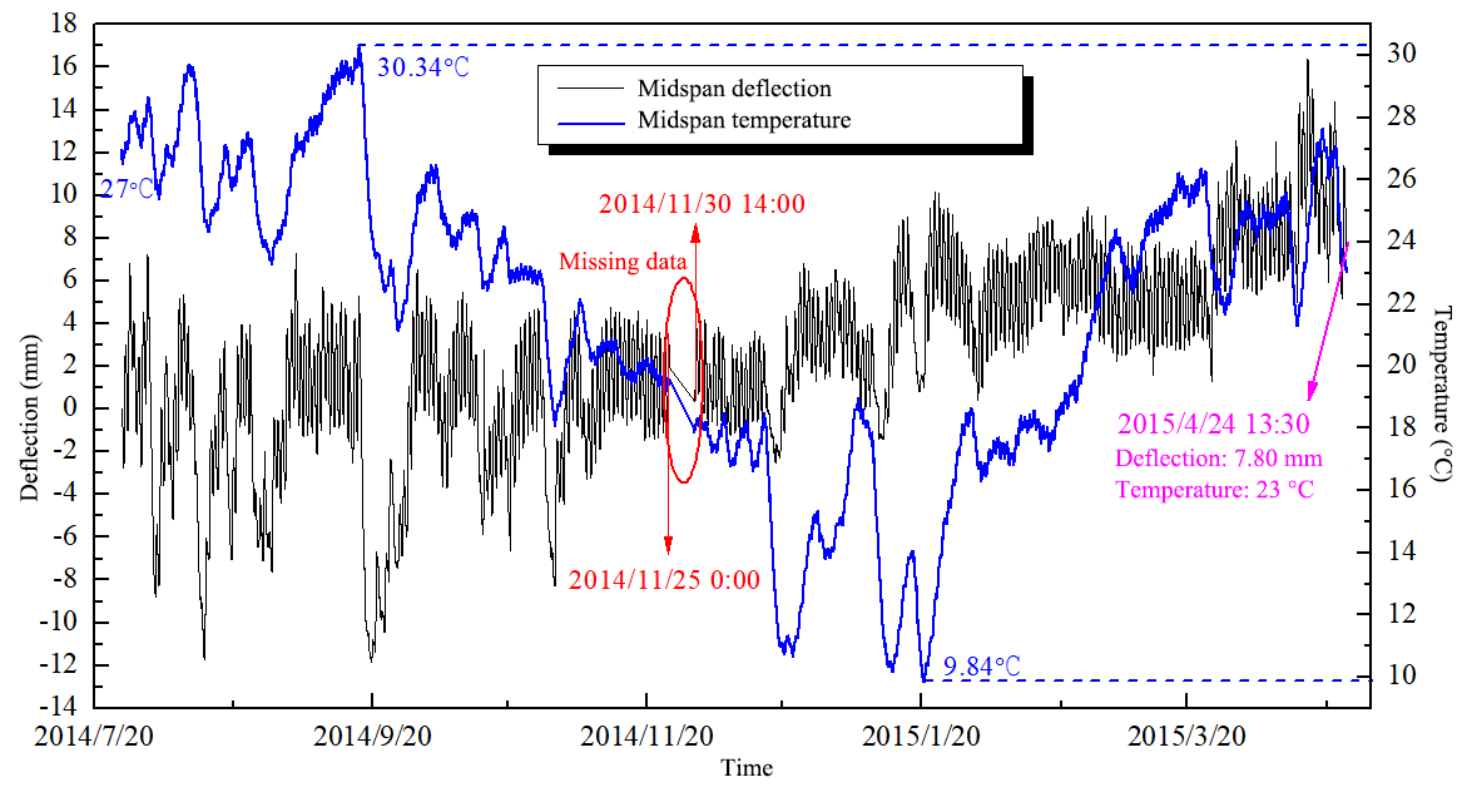

Figure 5. Time history of midspan defection and temperature 
As shown in Figures 4 and 5, the maximum, minimum, mean and instantaneous differences between the hydrostatic level and the reference value at the midspan of the right span were smaller than $1 \mathrm{~mm}$ every $10 \mathrm{~min}$. The trend of the mean value is the closest to the actual change in deflection of the bridge. To eliminate the interference of diurnal temperature, the monitoring data were collected in daytime in the research period. From Nov 10-14, 2014, the midspan temperature fluctuated between $19^{\circ} \mathrm{C}$ and $21^{\circ} \mathrm{C}$, while the bridge deflection did not change significantly, with a daily peak at $4-5 \mathrm{~mm}$. Moreover, the most significant up-warping occurred at 11:0012:00, and the most obvious down-warping appeared at 20:0021:00.

During the 9-month long system operation, data loss only appeared in Nov. 25-30, 2014. The fault was caused by the communicating line in the integrated control box, as shown in the box of Figure 2(c). After field maintenance, no more missing data was observed, revealing the good stability of the system. The initial difference of midspan temperature was $+4^{\circ}$ $\mathrm{C}$ and the measured deflection amounted to $7.8 \mathrm{~mm}$. The error is in line with the change law of bridge deflection. The deflection-temperature relationship is nonlinear. Slow changes of temperature may cause periodic deformation. However, the bridge will not warp up with rising temperature or warp down with falling temperature. The nonlinearity is more prominent when the temperature sags or swells, due to the lags in the temperature change of the bridge.

The raw monitoring data, plug the indices of MySQL database occupy, occupy roughly $120 \mathrm{MB}$ of space per month. Thus, the $23 \mathrm{~GB}$ hard disk can theoretically store 20 -year data. To ensure system performance, half of the raw data and indices were deleted every two years, and the disk space was optimized and partially released.

To sum up, the proposed integrated SHM system is applicable to actual bridges in remote regions, especially those with abundant sunshine and the basic $3 \mathrm{G} / 4 \mathrm{G}$ network. The system can operate smoothly to capture valid data, control the bridge site client, and support remote debugging. More importantly, the system operations only incurs a very low cost.

\section{CONCLUSIONS}

The stability and data validity are the focuses of the SHM system design for bridges. In this paper, an SHM system for bridges in remote regions is established based on opensource hardware rather than traditional industrial PC, Linux instead of Windows, and VPS in place of multiple physical servers.

In the system, different types of sensors are networked closely. Based on free opensource software, a 9-module software was designed under the original communication protocol and API for equipment. In this way, the bridge data can be collected in a synchronized manner. The raw data are filtered before uploading, such that only the valid data and fault alarms are transmitted to the monitoring center.

In addition, the dual PC redundancy was implemented to ensure the operating reliability of the system, due to its low price, high cost effectiveness and limited power consumption. The VPN and reverse SSH tunneling were introduced to enable the bridge site client to actively access the server side, allowing operators to remotely modify, debug and control the client side. To reduce the maintenance cost, the intelligent fault diagnosis was established to identify and report the abnormalities in system operation, such as surplus power supply and intrusion.

All in all, the proposed integrated SHM system is applicable to actual bridges in remote regions, especially those with abundant sunshine and the basic $3 \mathrm{G} / 4 \mathrm{G}$ network. The application results fully demonstrate the robustness, accuracy and cost effectiveness of the proposed system. This research fills up the gap in Linux-based SHM system design for bridges in remote regions, providing a reliable and advanced reference for the construction of bridge SHM systems.

\section{ACKNOWLEDGMENT}

This work was supported by the National Science Fund for Distinguished Young Scholars (51425801), The Science and Technology Project of Guizhou Provincial Transportation Department (2018-122-013).

\section{REFERENCES}

[1] Li, H., Zhou, W.S., Ou, J.P., Yang, Y. (2006). A study on system integration technique of intelligent monitoring systems for soundness of long-span bridges. China Civil Engineering Journal, 39(2): 46-52. https://doi.org/10.3321/j.issn:1000-131X.2006.02.010

[2] Bilodeau, M., Quaegebeur, N., Masson, P. (2017). Design of a guided wave absorber for structural health monitoring system development. NDT \& E International, 88: 33-41. https://doi.org/10.1016/j.ndteint.2017.03.003

[3] Liu, S.M., Zhang, L.L., Zhou, J.T. (2012). A new strategy for structural health monitoring based on structural destroyed mode and data correlation. Intelligent Automation \& Soft Computing, 18(6): 671678. https://doi.org/10.1080/10798587.2012.10643276

[4] Yang, J., Zhou, Y., Zhou, J., Chen, Y. (2013). Prediction of bridge monitoring information chaotic using time series theory by multi-step bp and RBF neural networks. Intelligent Automation \& Soft Computing, 19(3): 305314. https://doi.org/10.1080/10798587.2013.824161

[5] Martín, F.F., Llopis, M.V., Rodríguez, J.C., González, J.B., Blanco, J.M. (2014). Low-cost open-source multifunction data acquisition system for accurate measurements. Measurement, 55: 265-271. https://doi.org/10.1016/j.measurement.2014.05.010

[6] Klingensmith, B., Burgess, S.R., Campbell, T.A., Sherman, P.G., Buyukozturk, O. (2014). Modular system for high-speed 24-bit data acquisition of triaxial mems accelerometers for structural health monitoring research. Special Topics in Structural Dynamics, 6: 313-320. https://doi.org/10.1007/978-3-319-04729-4_26

[7] Chen, B., Wang, X., Sun, D., Xie, X. (2014). An integrated system for structural health monitoring and intelligent management for a cable-stayed bridge. The Scientific World Journal, 689471. http://dx.doi.org/10.1155/2014/689471

[8] Yang, J., Zhou, J. (2010). A dynamic life evolution method based on time-dependent reliability by health monitoring data. Intelligent Automation \& Soft Computing, 16(5): 645-651. https://xs.scihub.ltd/https://doi.org/10.1080/10798587.2 010.10643107

[9] Li, H., Ou, J.P. (2011). Structural health monitoring: From sensing technology stepping to health diagnosis. 
Procedia Engineering, 14: 753-760. https://doi.org/10.1016/j.proeng.2011.07.095

[10] Ko, J.M., Ni, Y.Q. (2005). Technology developments in structural health monitoring of large-scale bridges. Engineering Structures, 27(12): 1715-1725. https://doi.org/10.1016/j.engstruct.2005.02.021

[11] Lanata, F. (2015). Monitoring the long-term behaviour of timber structures. Journal of Civil Structural Health Monitoring, $5(2)$ : 167-182. https://doi.org/10.1007/s13349-014-0095-2

[12] Monnier, T., Guy, P., Lallart, M., Petit, L., Guyomar, D., Richard, C. (2008). Optimization of signal preprocessing for the integration of cost-effective local intelligence in wireless self-powered structural health monitoring. Advances in Science and Technology, 56: 459-468. https://doi.org/10.4028/www.scientific.net/AST.56.459

[13] Jung, H., Lee, K., Chun, W. (2006). Integration of GIS, GPS, and optimization technologies for the effective control of parcel delivery service. Computers \& Industrial Engineering, 51(1): 154-162. https://doi.org/10.1016/j.cie.2006.07.007

[14] Fraser, M., Elgamal, A., He, X., Conte, J.P. (2010). Sensor network for structural health monitoring of a highway bridge. Journal of Computing in Civil Engineering, 24(1): 11-24 https://doi.org/10.1061/(ASCE)CP.1943-5487.0000005

[15] Guan, H., Karbhari, V.M., Sikorsky, C.S. (2010). Webbased structural health monitoring of an FRP composite bridge. Computer-Aided Civil and Infrastructure Engineering, 21(1): 39-56. https://doi.org/10.1111/j.1467-8667.2005.00415.x

[16] Boller, C. (2000). Next generation structural health monitoring and its integration into aircraft design. International Journal of Systems Science, 31(11): 13331349. https://doi.org/10.1080/00207720050197730

[17] Zaurin, R., Catbas, F.N. (2009). Integration of computer imaging and sensor data for structural health monitoring of bridges. Smart Materials and Structures, 19(1):
015019. https://doi.org/10.1088/0964-1726/19/1/015019

[18] Ling, Y., Mahadevan, S. (2012). Integration of structural health monitoring and fatigue damage prognosis. Mechanical Systems and Signal Processing, 28: 89-104. https://doi.org/10.1016/j.ymssp.2011.10.001

[19] King, R.C., Villeneuve, E., White, R.J., Sherratt, R.S., Holderbaum, W., Harwin, W.S. (2017). Application of data fusion techniques and technologies for wearable health monitoring. Medical Engineering \& Physics, 42: 1-12. https://doi.org/10.1016/j.medengphy.2016.12.011

[20] Daroogheh, N., Baniamerian, A., Meskin, N., Khorasani, K. (2016). Prognosis and health monitoring of nonlinear systems using a hybrid scheme through integration of PFS and neural networks. IEEE Transactions on Systems, Man, and Cybernetics: Systems, 47(8): 1990-2004. https://doi.org/10.1109/TSMC.2016.2597272

[21] Bae, S.C., Jang, W.S., Woo, S., Shin, D.H. (2013). Prediction of WSN placement for bridge health monitoring based on material characteristics. Automation in Construction, 35: 18-27. https://doi.org/10.1016/j.autcon.2013.02.002

[22] Wang, C.M. (2011). New application of wireless sensor network in bridge-health monitoring. Computer \& Modernization, $\quad 1$ : $145-148, \quad 154$. https://doi.org/10.3969/j.issn.1006-2475.2011.01.042

[23] Vanzwol, T.R., Cheng, J.J.R., Tadros, G. (2008). Longterm structural health monitoring of the crowchild trail bridge. Canadian Journal of Civil Engineering, 35(2): 179-189. https://doi.org/10.1139/L07-073

[24] Ogundipe, O., Lee, J. K., Roberts, G. W. (2014). Wavelet de-noising of GNSS based bridge health monitoring data. Journal of Applied Geodesy, 8(4): 273-282. https://doi.org/10.1515/jag-2014-0011

[25] Venglar, M., Sokol, M., Aroch, R., Budaj, J. (2016). Initial experimental test of the port bridge for structural health monitoring. Applied Mechanics \& Materials, 837: 135-139. https://doi.org/10.4028/www.scientific.net/AMM.837.1 35 